\title{
The Untargeted Capability of NMR Helps Recognizing Nefarious Adulteration in Natural Products
}

Seon Beom Kim, Jonathan Bisson, J. Brent Friesen, Luca Bucchini, Stefan Gafner, David C. Lankin, Shao-Nong Chen, Guido F. Pauli, ${ }^{*}$ and James B. McAlpine* 


\section{TABLE OF CONTENTS}

Figure S1. Non-polar extraction workflow of the investigated two batches of S-3 curcumin dietary supplements.

Figure S2. Polar extraction workflow of the investigated two batches of curcumin S-4 dietary supplements.

Figure S3. The dried non-polar and polar solvent extracts from the investigated S-5 curcumin dietary supplements.

Figure S4. UHPLC-UV chemical profiling of the investigated curcumin dietary S-6 supplements and five authentic reference compounds.

Figure S5. LC analysis of impurities in the non-polar extracts of the investigated S-7 curcumin dietary supplements.

Figure S6. The ${ }^{1} \mathrm{H}$ NMR chemical fingerprints of the investigated curcumin dietary S-8 supplements. 
Figure S1. Non-polar extraction workflow of the investigated two batches of curcumin dietary supplements.

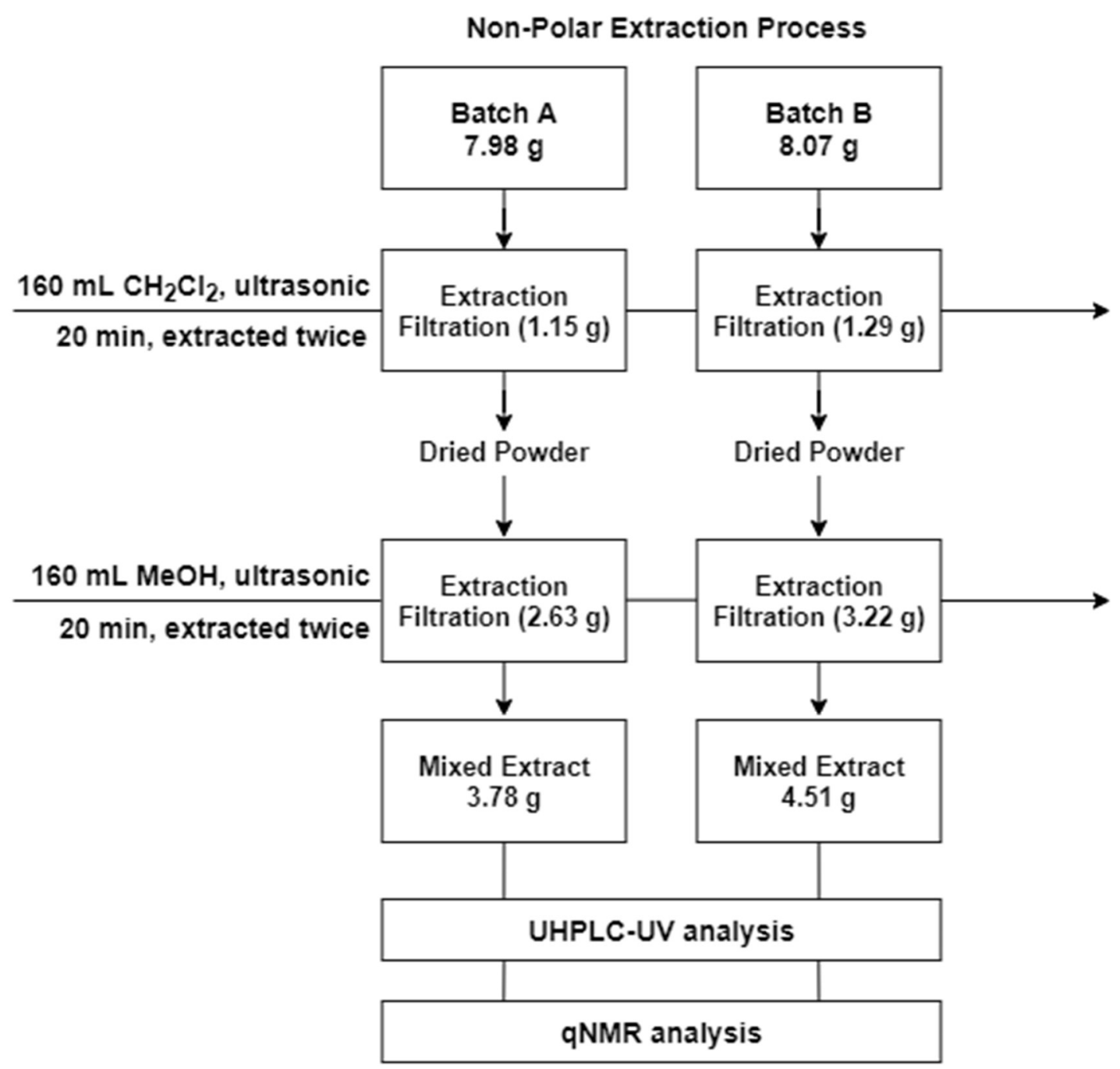

Ten tablets from each of batched A and B were prepared for the non-polar extraction process. Extracted twice with sonication and maceration using two different solvent systems, $\mathrm{CH}_{2} \mathrm{Cl}_{2}(20: 1$, $\mathrm{v} / \mathrm{w})$ and $\mathrm{MeOH}(20: 1, \mathrm{v} / \mathrm{w})$. The combined extracts were utilized for the identification of chemical profile and phytochemical fingerprint using UHPLC-UV and qNMR analysis techniques. 
Figure S2. Polar extraction workflow of the investigated two batches of curcumin dietary supplements.

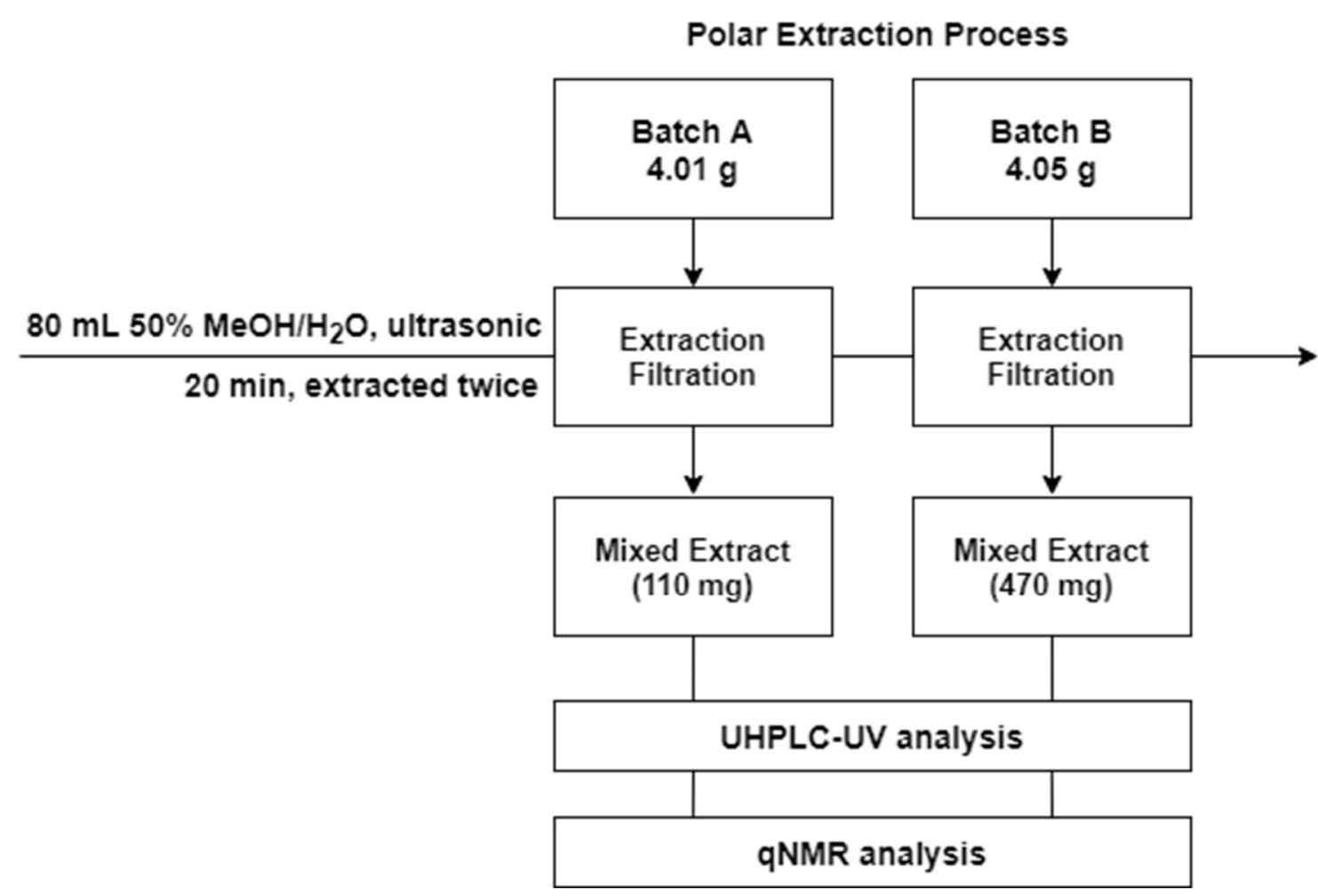

Five tablets of batch A and B were prepared for the polar extraction process. Extracted twice with sonication and maceration using $50 \% \mathrm{MeOH} / \mathrm{H}_{2} \mathrm{O}(20: 1, \mathrm{v} / \mathrm{w})$. The extracts were utilized for the identification of chemical profile and phytochemical fingerprint using (U)HPLC-UV and qNMR analysis techniques. 
Figure S3. The dried non-polar and polar solvent extracts from the investigated curcumin dietary supplements.

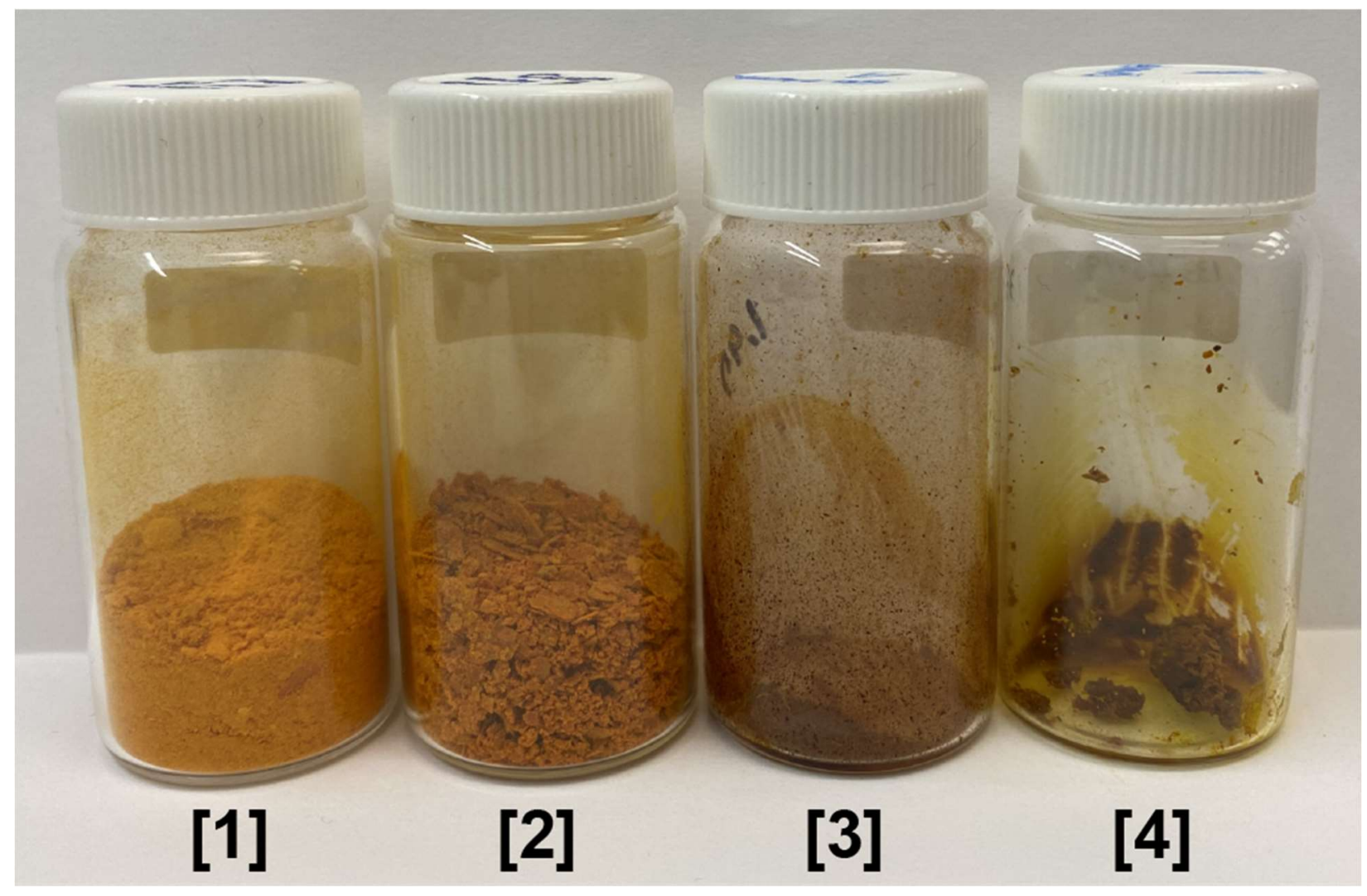

Extracts of curcumin dietary supplement samples batch A and B show significant color and yield differences. The non-polar solvent extract obtained weighed $3.78 \mathrm{~g} \mathrm{(47.6 \% )}$ for batch A [1] and $4.51 \mathrm{~g}(52.9 \%)$ for batch B [2]. The polar solvent extracts confirmed batch disparity: $110 \mathrm{mg}$ (2.74\%) were obtained for batch A [3] and $470 \mathrm{mg}(11.60 \%)$ for batch B [4]. 
Figure S4. UHPLC-UV chemical profiling of the investigated curcumin dietary supplements and five authentic reference compounds.

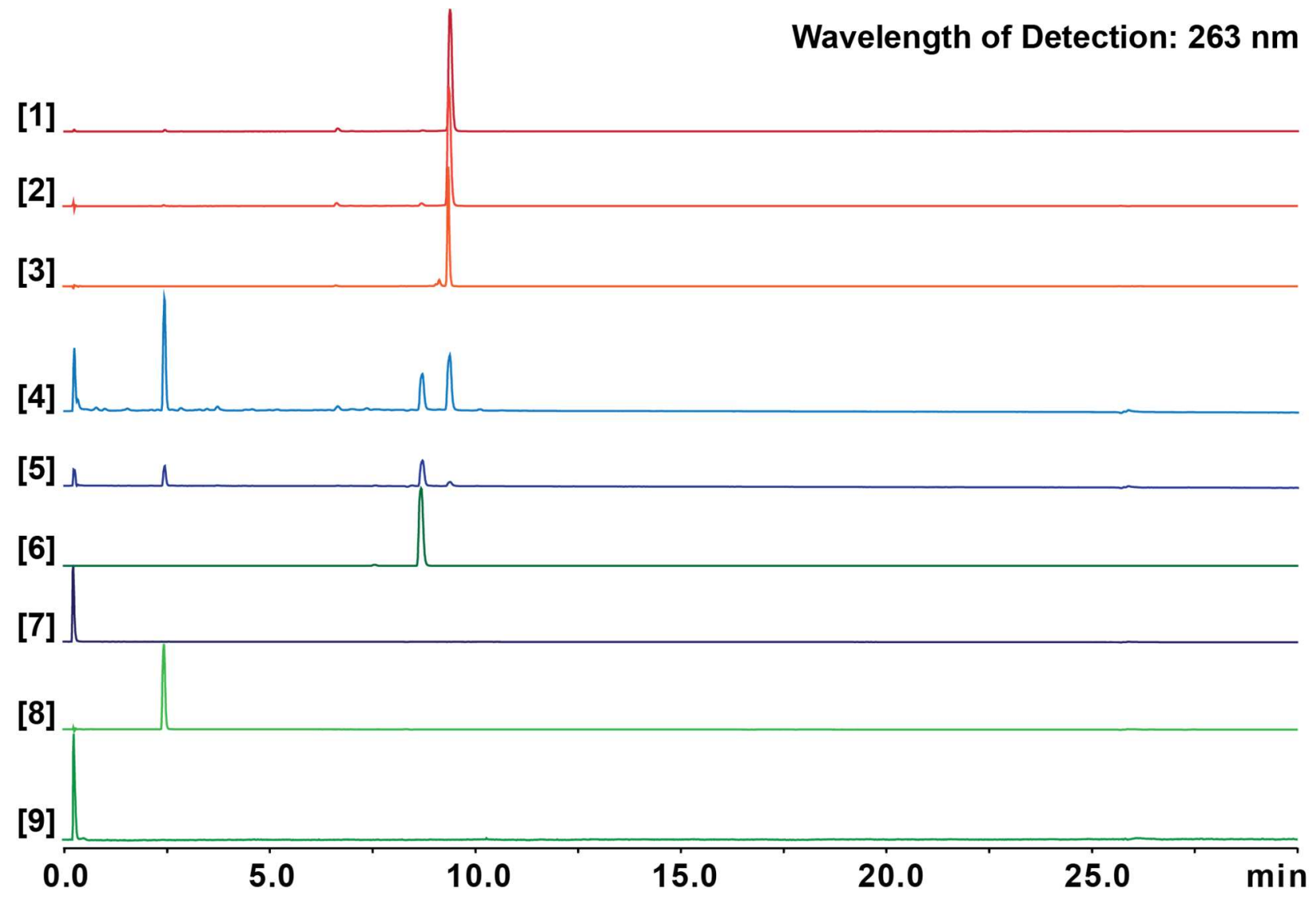

Stacked UHPLC-UV chromatograms (detected: $263 \mathrm{~nm}$ ) of curcumin dietary supplement extracts: batches A [1 and 4] and B [2 and 5] extracted with $100 \% \mathrm{CH}_{2} \mathrm{Cl}_{2}$ and $100 \% \mathrm{MeOH}$ and $[50 \%$ $\mathrm{MeOH} / \mathrm{H}_{2} \mathrm{O}$ ), respectively. Reference compounds were: curcumin [3], piperine [6], vitamins B1 (thiamine) [7], B2 (rboflavin) [8] and B6 (pyridoxine) [9]. 
Figure S5. UHPLC analysis of the non-polar extracts of the investigated curcumin dietary supplements.

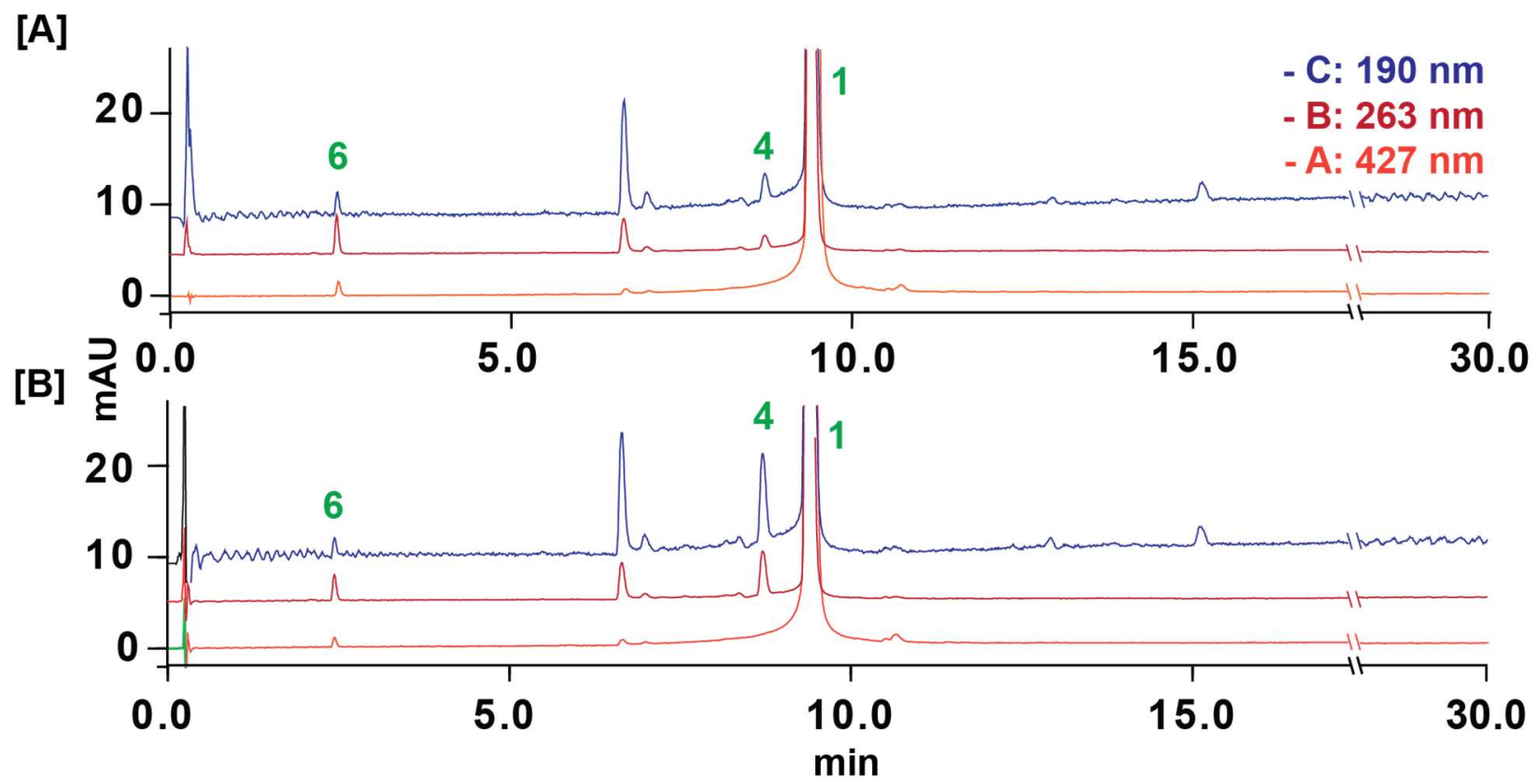

UHPLC-UV analysis of the non-polar extracts of batches A [A] and B [B] using three different UV detection wavelengths. Curcumin (1), piperine (4) and riboflavin (6) were identified by retention time and their UV spectra. While the results confirmed the absence of natural curcuminoid constituents in the extracts of the designated "curcumin" dietary supplement, other unidentified residual peaks were observed. 
Figure S6. The ${ }^{1} \mathrm{H}$ NMR chemical fingerprints of the investigated curcumin dietary supplements.

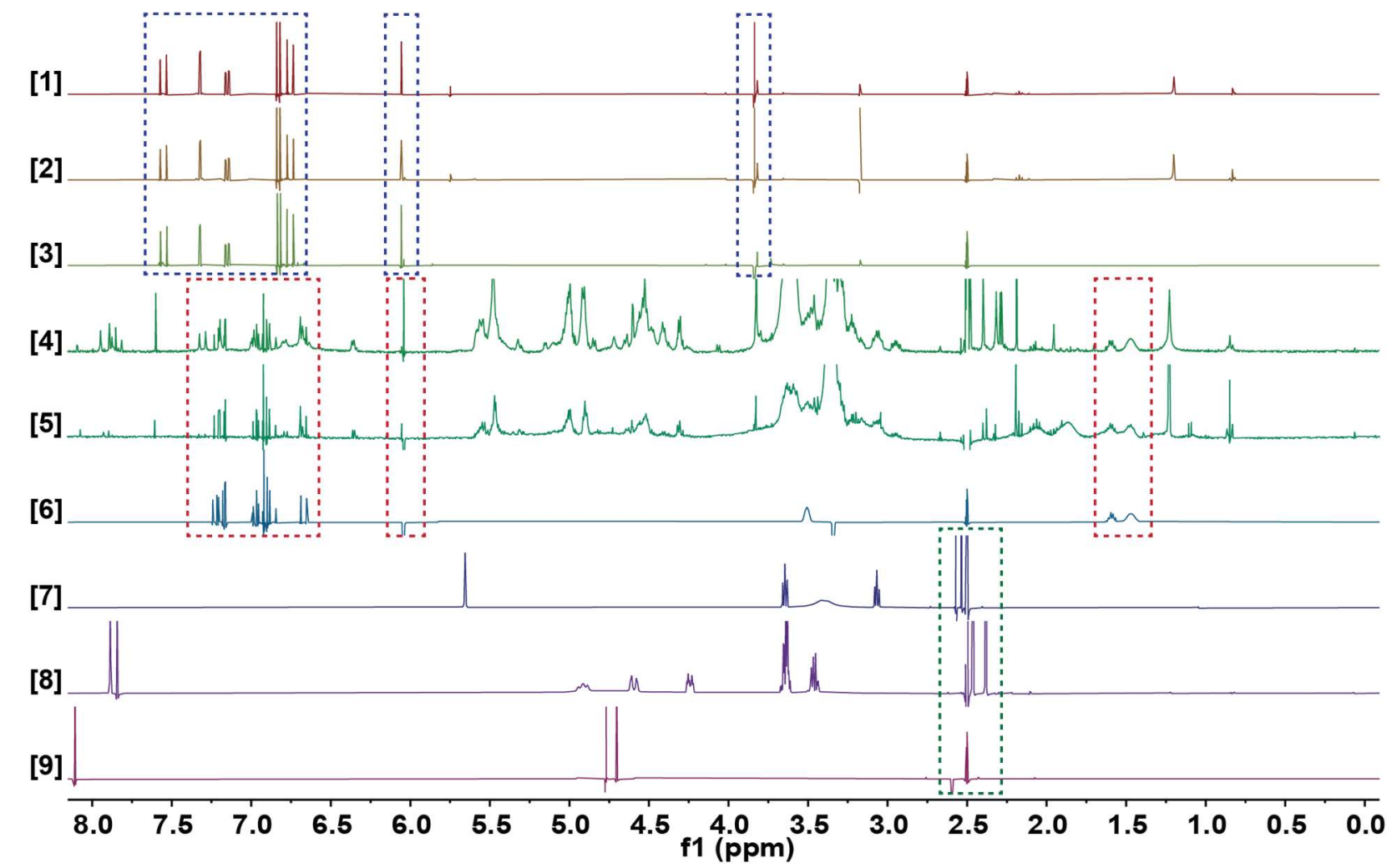

Stacked ${ }^{1} \mathrm{H}$ NMR (DMSO- $d_{6}, 400 \mathrm{MHz}$ ) spectra of curcumin dietary supplement extracts: batches $\mathrm{A}$ [1 and 4] and $\mathrm{B}$ [2 and 5] extracted with $\left[100 \% \mathrm{CH}_{2} \mathrm{Cl}_{2}\right.$ and $\left.100 \% \mathrm{MeOH}\right]$ and $[50 \%$ $\mathrm{MeOH} / \mathrm{H}_{2} \mathrm{O}$ ], respectively. Reference compounds were: curcumin in trace [3], piperine in trace [6], vitamins B1 (thiamine) in trace [7], B2 (riboflavin) in trace [8], and B6 (pyridoxine) in trace [9]. 